\title{
Avoiding errors attributable to topography in GPS-IR snow depth retrievals
}

\author{
Shuangcheng Zhang, Xiaolei Wang* and Qin Zhang \\ College of Geology Engineering and Geomantic, Chang'an University, Xi'an, \\ Shanxi 710054, China \\ *Corresponding author: Xiaolei Wang (chd_wxl@qq.com)
}

\begin{abstract}
Global Positioning System (GPS) Interferometric reflectometry represents a potential source of new snow data for climate scientists and water managers with spatial and temporal sensitivity. Generally, the snow layer fluctuation is considered to be correlated with the ground surface fluctuation. The reflector heights in terms of the signal-to-noise ratio (SNR) are quite close to the vertical distance between the antenna and the ground or snow level at the corresponding Fresnel zone. The reflector heights at different zones were represented by a grid model in this work, which can reflect and overcome some of the problems caused by the topography. The proposed method for snow depth retrievals looks for good quality reflector height values of the horizontal reflecting zone in the grid model, and with this method improvements in snow depth retrieval accuracy were achieved (RMSE: $7.40 \mathrm{~cm}$, Corr.: 0.99) compared to the PBO H2O group calculation results (RMSE: $16.58 \mathrm{~cm}$, Corr.: 0.99).
\end{abstract}

Keywords: GPS-IR; Snow depth retrievals; Surface fluctuation

\section{Introduction}

With ongoing research and applications involving the Global Positioning System (GPS), evidence of multipath errors has gradually emerged. When the radio navigation signals of GPS reflect off the surface, the reflected GPS signals contain information about the surface and subsurface. Specifically, the analysis of the waveform, polarization, amplitude, phase, frequency, and other parameters of GPS reflected signals effectively allows one to acquire the physical characteristics of the surface and subsurface. This has become a popular remote sensing technology, termed GPS Reflectometry (GPS-R).

The use of ground-reflected signals from navigation satellites to remotely sense and study environmental variables was first proposed by Hall and Cordey (1988), who determined the surface wind velocity over the ocean. More recent studies have used GPS ground reflections to estimate land surface parameters including snow depth (Larson et al., 2009; Larson and Nievinski, 2013), soil moisture (Grant et al., 2007; Katzberg et al., 2006; Larson et al., 2008a, 2008b), and vegetation phenology 
(Rodriguez-Alvarez et al., 2012b). This technique, known as GPS-Interferometric Reflectometry (GPS-IR), relates temporal changes in signal-to-noise ratio (SNR) interferograms to changes in environmental parameters surrounding a GPS antenna for an area that scales with the height (Nievinski, 2013).

At present, certain valuable results have been obtained with GPS-IR technology. Bilich et al. (2004) studied the separation of direct and reflected signals within the SNR, and the relationship between the environment and the reflected signal was assessed. Larson et al. (2008a, 2008b, 2009) and Larson and Nievinski (2013) refined the GPS-IR technology by using SNR observations to retrieve snow depth and soil moisture. Jacobson (2010) discussed the special case of a snow-covered frozen lake and explored the potential for inferring lake ice thickness based on a case study. Ozeki and Heki (2011) presented an algorithm for snow depth detection based on the L4 carrier-phase linear combination and obtained results mostly consistent with the SNR. Yu et al. (2015) proposed a new snow depth estimation approach that uses a geodetic GPS station, multipath reflectometry, and a linear combination of phase measurements consisting of GPS triple-frequency (L1, L2, and L5) signals; specifically, they established analytical linear models to determine snow depth. Rodriguez-Alvarez et al. (2012a) analyzed snow effects by using the interference pattern technique (IPT) and developed a retrieval algorithm for snow-covered soil surfaces. Jin and Najibi (2014) performed retrieval research on snow depth, snow layer temperature, and other characteristic parameters of the terrain surface by using GPS combined with geometric-free distance L4 carrier-phase observations. Nievinski and Larson (2014a, 2014b) offered a forward/inverse approach for GPS-IR retrieval of snow depth, and the formulation, simulation, application, and validation of the inverse modeling of the GPS multipath was analyzed. Boniface et al. (2015) compared gridded snow depth estimates from the Snow Data Assimilation System (SNODAS) with snow depth observations derived from GPS-IR. The GPS-IR estimates of snow depth were shown to provide useful error characterization of SNODAS products across much of the western United States, and they were considered to have potential as an additional data assimilation source that could improve SNODAS estimates.

In the process of snow depth retrievals, it is important not to overlook the complicated influence of the topographical environment. Different satellite signals transmitted from different azimuth and elevation angles are reflected by a certain area on the ground. Thus, the reflected signal, received by a GPS receiver, is inevitably affected by the terrain of this specific area. This paper analyzes the influence of ground deviations on snow depth retrievals through the application of a grid model.

\section{Background}

The GPS radio navigation signals of different azimuths reflected from special surface zones carry characteristic information for that reflecting zone. In other words, every signal intercepted has a Fresnel zone and distinct "SNR metrics"- the 
reflector height $R H$ is focused on in this study. Here, we review a number of concepts and principles related to snow depth retrievals.

A. SNR: This is a measure of the signal strength received by a GPS antenna. It is a consequence primarily of the direct or line-of-sight power $\left(P_{d}\right)$ and secondarily of the reflected power $\left(P_{r}\right)$ (Larson and Nievinski, 2013):

$$
S N R \propto P_{d}+P_{r}+2 \sqrt{P_{d} P_{r}} \cos \phi .
$$

The SNR is determined by the transmitted power of the satellite, by the antenna gain pattern, and by whether the receiver uses code-enabled tracking methods. Superimposed on this trend are modulations at the rising or setting ends of the arc; these peaks and valleys are constructive and destructive interference, and they mainly depend on the phase difference $(\phi)$ between direct and reflected signals (Zavorotny et al., 2010).

B. Principles of snow depth retrievals: The main principles were proposed by Larson et al. (2009) and Larson and Nievinski (2013). When a signal from a satellite reaches the receiver antenna, both the direct and the reflected signals are included. Compared with the direct signal, the reflected signal propagates an additional distance (i.e., it is delayed), which can be described as D = (D1 + D2), as shown in Fig. 1.

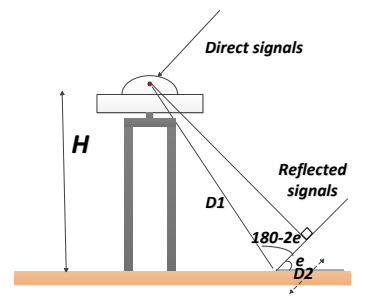

Fig. 1. Simple reflection model. Symbol $e$ is the elevation angle (with respect to the horizon).

From Fig. 1:

$$
\begin{aligned}
& D_{1}=D_{2} \cos (\pi-2 e), \\
& D_{2}=H / \sin (e), \\
& D=2 H \sin (e) .
\end{aligned}
$$

At this point, the phase difference between the direct and reflected signals is:

$$
\phi=2 \pi D / \lambda=4 \pi H \sin (e) / \lambda \text {. }
$$

Therefore,

$$
\frac{d \phi}{d \sin (e)}=4 \pi \frac{H}{\lambda} .
$$

In Eq. (6), under the one-ray reflection condition, parameter $\phi$ has a linear relationship with $\sin (e)$. It is known from Eq. (1) that the SNR observation has a sinusoidal relationship with the relative phase $\phi$. Therefore, the SNR and sine 
can approximately present a sinusoidal relationship at a certain frequency. In the theory of Larson et al. (2010, 2013), this frequency is acquired by Lomb-Scargle (L-S) spectrum analysis applied to the detrended SNR. From Eq. (6), the sinusoid frequency can be expressed by $f=\frac{2 H}{\lambda}$. Here, parameter $H$ is the vertical distance between the antenna phase center and the snow surface, which we call the reflector height $R H$.

Larson and Nievinski (2013) proposed the detailed steps used to obtain the RH. The figures of the intermediate steps of the processing are shown in Fig. 2.
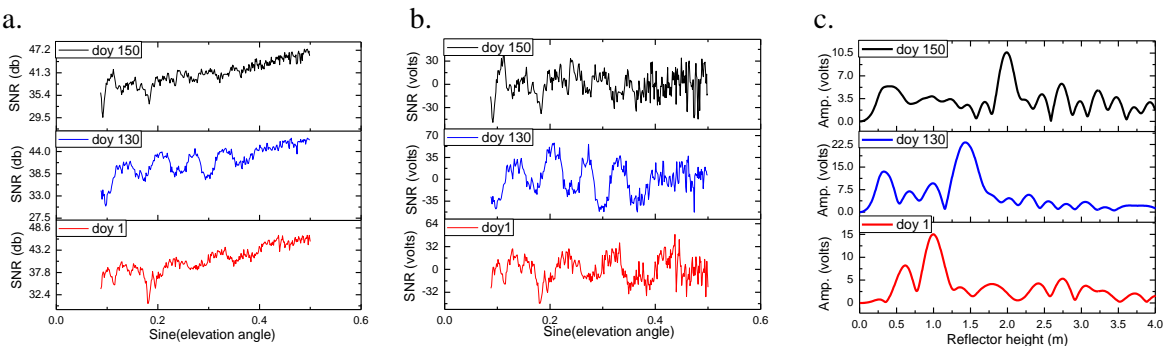

Fig. 2. a. Recorded SNR L1 C/A data for PRN 19 at PBO receiver p351 between elevation angles of $5^{\circ}$ and $30^{\circ}$ on doy 1,130 , and 150 for the year 2015. b. Rising arc of PRN 19 SNR data, with the second-order polynomial removed and data converted from $\mathrm{dB}$ to linear units (for simplicity, volts). c. Lomb-Scargle periodograms computed for the SNR data.

Fig. 2 shows an example of how RH can be obtained from GPS SNR data. The changes in reflector height are pronounced for this satellite track, and the peaks are much greater than the background Lomb-Scargle periodograms noise, thus indicating a strong specular reflection. The RH values corresponding to the peaks on doy (day of year) 1,130 , and 150 were $1.0 \mathrm{~m}, 1.5 \mathrm{~m}$, and $2.0 \mathrm{~m}$, respectively.

C. First Fresnel zone (FFZ) (e.g., Hristov, 2000; Larson and Nievinski, 2013; see also the Appendix): The coherent reflection from the GPS footprint can be derived from the bistatic radar literature as the FFZ. For a nominal horizontal surface, it fundamentally depends on the height of the antenna above the reflecting surface and on the elevation angle between the satellite and the local horizon at the antenna, as shown in Fig. 3.

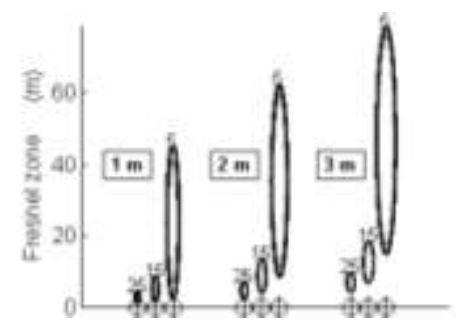

Fig. 3. The FFZ varies with the height of the antenna and the elevation angle. The circle containing a cross is the antenna position. The number in the box is the height of the antenna. The number at the top of the ellipse is the elevation angle, after Larson and Nievinski (2013). 
Fig. 3 reveals that the FFZ becomes larger with increases in the antenna height and decreases in the elevation angle. Furthermore, the center point of the FFZ becomes closer to the antenna with decreases in the antenna height and increases in the elevation angle.

D. Snow depth: Snow depth is defined by Larson and Nievinski (2013) as follows:

Snow depth $=($ vertical distance to the ground $)-($ vertical distance to the snow layer).

The first term in Eq. (7) represents the vertical distance between the antenna and the sensed ground. However, this cannot be assumed to be equal to the height of the antenna above the ground that is immediately below because in reality, it will be greater for a downhill slope and smaller for an uphill slope. In practice, SNR data can be used to estimate the vertical distance to the ground (i.e., the $R H$ of bare-ground) in a similar manner to which estimations of the distance to the snow layer can be made. Snow depth $(S D)$ can be calculated in an equivalent form:

$$
S D=R H_{G}-R H_{S},
$$

where $R H_{G}$ is the reflector height of the ground underlying the snow reflection and $R H_{S}$ is the reflector height of the snow overlying the ground reflection.

\section{Influence of ground fluctuations on RH retrievals}

The planar information of a reflection area can be derived by interpolating the SNR RH of reflected signals according to the coordinates of the corresponding reflection zone. Here, we review a number of concepts and principles related to snow depth retrievals.

When there are a series of $N$ SNR data points left for retrieving the planar parameter after being intercepted by the phase and elevation angle, the SNR data are presented as $\{S N R\}_{N}=\left\{S N R_{1}, S N R_{2}, \cdots, S N R_{n}, \cdots, S N R_{N}\right\}$, where the second index refers to the each data point within the SNR series. Every signal intercepted has its FFZ and a value for RH, and each FFZ corresponds to each scenario. For an effective SNR, the effective reflection area is considered to be the integrated FFZ of the mean of the selected elevation angles. The $F F Z_{n}$ of $S N R_{n}$ is shown Fig. 4 and corresponds to $R H_{n}$. The focal points of $F F Z_{n}$ are $C_{1_{n}}$ and $C_{2_{n}}$, with coordinates presented as $\left(x_{C_{1_{n}}}, y_{C_{1_{n}}}\right)$ and $\left(x_{C_{2}}, y_{C_{2 n}}\right)$, respectively, and these are shown as red dots in Fig. 4; the major axis length is $2 a_{\mathrm{n}}$, and the focal length is $2 c_{n}$. 


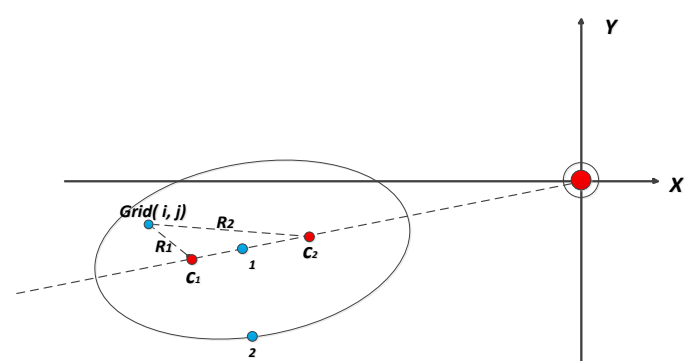

Fig. 4. Principles of the snow depth grid model.

In this paper, a grid model is used to represent the planar parameter value for a certain point at $\operatorname{grid}(i, j)$ with coordinate $\left(x_{i}, y_{j}\right)$. The corresponding value of $R H$ in this grid mode is:

$$
R H(i, j)=\frac{1}{\sum_{n=1}^{N} \omega_{n}} \sum_{n=1}^{N} w_{n} \cdot R H_{n}
$$

Here, $w_{n}$ is the weight of $R H_{n}$ with respect to $\operatorname{grid}(i, j)$. In this work, when $\operatorname{grid}(i, j)$ is on the center of $F F Z_{n}$, the weight is considered to be 1 ; when $\operatorname{grid}(i, j)$ is on the border line of $F F Z_{n}$, it is considered to be $S$ (a given threshold value). Also, the weight should be inversely proportional to the distance. In this way, the weight is defined as follows:

$$
w_{n}=1-\frac{1-S}{2 a_{n}-2 c_{n}}\left(R-2 c_{n}\right),\left(0<S<1, w_{n}>0\right),
$$

where $S$ is the given threshold; it shows when $\operatorname{grid}(i, j)$ is on the border line of $F F Z_{n}, w_{n}=S . R$ is the sum of the distances between $\operatorname{grid}(i, j)$ and the focal points:

$$
\begin{aligned}
& R=R_{1}+R_{2} \\
& =\sqrt{\left(x_{i}-x_{C_{1_{n}}}\right)^{2}+\left(y_{j}-y_{C_{1_{n}}}\right)^{2}}+\sqrt{\left(x_{i}-x_{C_{2_{n}}}\right)^{2}+\left(y_{j}-y_{C_{2_{n}}}\right)^{2}} .
\end{aligned}
$$

In this paper, horizontal coordinates are transformed from Cartesian to polar coordinates for the calculation:

$$
\left\{\begin{array}{c}
\rho_{i}=\sqrt{x_{i}^{2}+y_{j}^{2}} \\
\theta_{j}=\arctan \frac{y_{j}}{x_{i}}
\end{array} .\right.
$$

In consideration of Eq. (9), Eq. (8) can be rewritten to:

$$
\begin{aligned}
& S D(i, j)=R H_{G}(i, j)-R H_{S}(i, j) \\
& \quad=\frac{1}{\sum_{n_{G} N_{G}}^{N_{1} w_{n_{G}}}} \sum_{n_{G}=1}^{N_{G}} w_{n_{G}} \cdot R H_{n_{G}}-\frac{1}{\sum_{n_{S}=1}^{N_{S}} w_{n_{S}}} \sum_{n_{S}=1}^{N_{S}} w_{n_{S}} \cdot R H_{n_{S}} .
\end{aligned}
$$

The grid values of the snow depth retrieval grid model $(S D(i, j))$ are equal to the $R H$ values of the bare-ground grid model $\left(R H_{G}(i, j)\right)$ minus the $R H$ values of the snow-covered grid model $\left(R H_{S}(i, j)\right)$. The parameters with the subscript of $G$ 
represent the corresponding parameters when the ground is bare, while the parameters with the subscript of $S$ represent the corresponding parameters with snow cover.

\section{Site description}

Snow can be monitored in a variety of ways. The Snowpack Telemetry (SNOTEL) network (Serreze et al., 1999) measures SWE (snow water equivalent) by using fluid-filled steel pillows and, more recently, snow depth has been measured by using in situ measurements. The GPS reflections research group PBO H2O use GPS reflection data from the National Science Foundation's (NSF's) Plate Boundary Observatory to study the water cycle, and they have published retrieval results for snow, vegetation, and soil moisture (http://xenon.colorado.edu/portal/). Therefore, the snow depth measured by SNOTEL and the PBO H2O group were used as references in this work.

The p351 EarthScope Plate Boundary Observatory (http://pbo.unavco.org) GPS site was chosen as the study site. The distance between p351 and the nearest SNOTEL site was $15.1 \mathrm{~km}$. We calculated the $R H$ of the SNR between doy 170 and 260 in 2015 (without snow and ice cover present) following Eq. (9) for the $R H$ grid model of bare-ground $\left(R H_{G}\right)$, as shown in Fig. $5 \mathrm{c}$.

a.

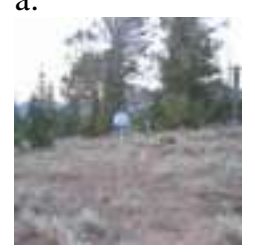

Site North

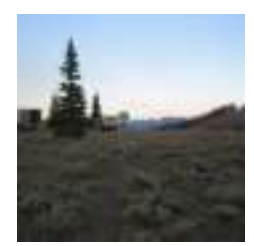

Site East

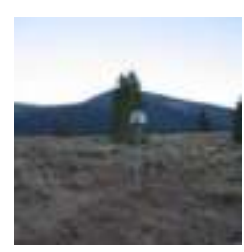

Site South

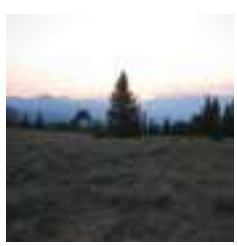

Site West

b.

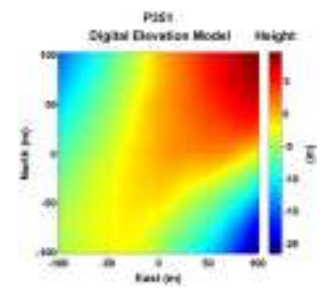

c.

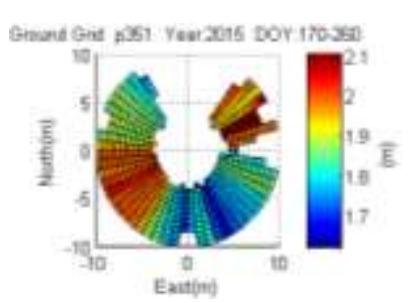

Fig. 5. a. Local environment around the $\mathrm{p} 351$ station at the study site (http://www.unavco.org/instrumentation/networks/status/pbo/photos/P351). b. Digital elevation model (DEM) diagram of p351 (the value at the center of the DEM is $0 \mathrm{~m}$, http://pbo.unavco.org). c. $H_{\text {eff }}$ grid model of bare-ground for the p351 station (units: meters). North increases to the top, and east increases to the right. The radius is $10 \mathrm{~m}$ based on the antenna phase center. When a grid cell is not occupied by any FFZ, its $R H$ value is left undefined, but not zero; these areas are shown in white.

Given the same surface features (the same soil and vegetation type) in each direction and without shading from trees and buildings around p351 (Fig. 5a), the 
surface slope characteristics around p351 can be easily extracted from Fig. 5b. The data shown in Fig. 5c illustrates the $R H$ grid model of bare-ground for the p351 station, and the values of $R H$ varied in the range of 1.6 to $2.1 \mathrm{~m}$.

As shown in Fig. 5b, the southwest areas of station p351 tended to be horizontally uniform, while the southeast and northwest areas of this station demonstrated a low-lying pattern, and the northeast areas displayed an obvious high-lying pattern. However, it is important to note that the scale of DEM used (200 $\mathrm{m} \times 200 \mathrm{~m}$ ) does not correspond to the current FFZ. Fortunately, pictures in four directions could be acquired, as shown in Fig. 5a; these pictures depict the same terrain as that shown in Fig. 5b. Thus, the lack of matched DEM data did not hinder the analysis. In Fig. 5c, the southwest regions showed uniform $R H$ values of $2 \mathrm{~m}$, while the northwest and southeast regions were marked with a cool color that is indicative of a smaller $R H$. Because there is a shed 30-m away due northeast of p351 and a tree immediately east, gross errors (abrupt color changes) appeared in the northeast area, and some grids in the east were left undefined owing to blocking reflections.

In summary, different terrains corresponded to different planar grid values of $R H$, which means that it was the topography that was really biasing the reflector height; thus, this issue can influence the snow depth retrievals.

\section{Inclusion of a constraint to avoid errors attributable to topography}

Theoretically, the simplest way to correct the errors caused by topography would be to choose an azimuth area that is near horizontal. Choosing reflector height values close to the station height can avoid the gross errors, and these will tend to correspond to the horizontal area, which enables the selection of an azimuth region devoid of sloping terrain to avoid ensuing errors. For example, if the station height is $2 \mathrm{~m}$, the following constraint will be included in Eq. (13):

$$
\left\{\begin{array}{l}
R H_{n_{G}} \in[1.5,2.5] \\
R H_{n_{S}} \in[0.5,2.5]
\end{array} .\right.
$$

Then, we calculated the snow depth retrieval grid model following Eq. (13) on doy 1, 50, 100, and 150 for year 2015. The grid is updated daily, as shown in Fig. 6.

a

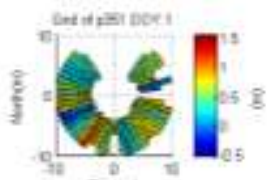

b

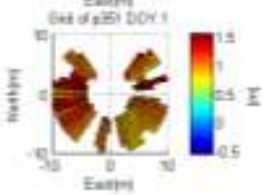

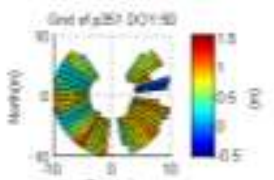

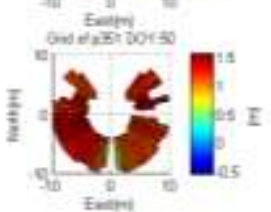

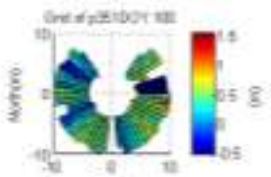

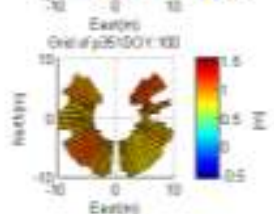

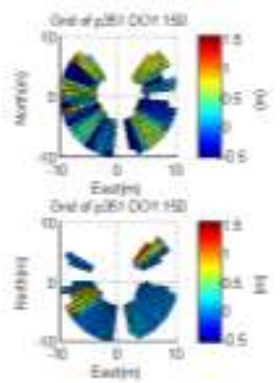


Fig. 6. Grid of p351. a. The snow depth retrieval grid model without constraint on doy 1, 50, 100 , and 150 of the year 2015 . b. The snow depth retrieval grid model with constraint on doy $1,50,100$, and 150 of the year 2015. (Because the signals were not acquirable in some azimuth areas, as well as the constraint, the polar diagram in Fig. 6 is incomplete.)

After adding constraint for $R H$, the color trend in Fig. $6 \mathrm{~b}$ became more obvious, i.e., red-and-orange in doy 1 , red in doy 50 , red-and-orange in doy 100 , and blue in doy 150. However, it is difficult to see the trend in Fig. 6a.

Averages were calculated by integrating over all the valid snow depth grid elements, and the averages were considered to be the snow depth retrieval results to avoid the errors caused by topography. In this way, this work calculated the series of snow depth grid model data and snow depth retrievals between doy 1 and doy 150 for the year 2015, as shown in Fig. 7.

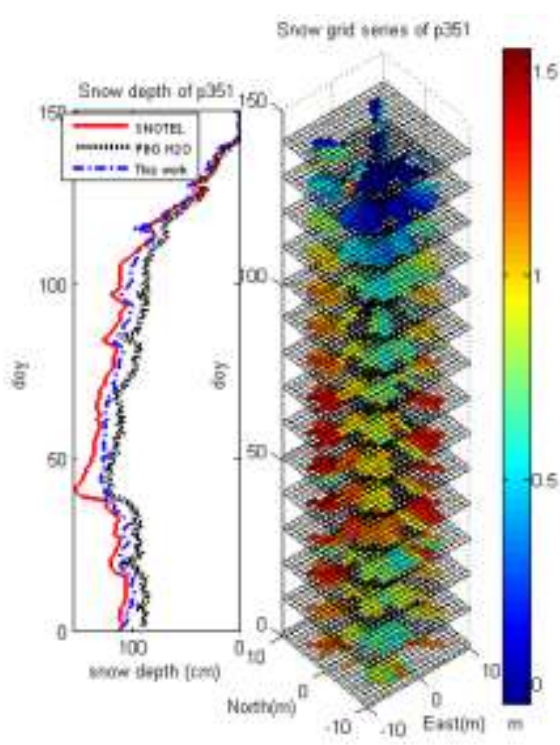

Fig. 7. The snow depth retrieval results of p351 with constraint.
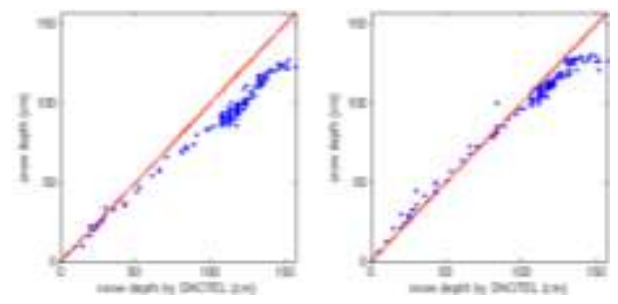

Fig. 8 Snow depth retrievals versus snow depth measured by SNOTEL (left: PBO H2O group results; right: this work).

Table 1. RMSEs and correlation coefficients for the p351 station; the data shown below were compared to SNOTEL data for this analysis.

\begin{tabular}{l|l|l}
\hline & PBO H2O & This work \\
\hline RMSE $(\mathrm{cm})$ & 16.58 & 7.40 \\
\hline $\begin{array}{l}\text { Correlation } \\
\text { coefficient }\end{array}$ & 0.99 & 0.99 \\
\hline
\end{tabular}

In the left sub-picture of Fig. 7, the red solid line indicates the snow depth measured by SNOTEL, the black dotted line represents the data for snow depth retrievals by the $\mathrm{PBO} \mathrm{H} 2 \mathrm{O}$ group, and the blue dot-and-dash line is the retrieval results of this study after selecting grids with a near-horizontal area. It can be seen that these three results follow the same trend, which means the SNR pattern is obvious for the snow depth changes. The results also show that this study achieved better snow depth retrievals than the $\mathrm{PBO} \mathrm{H} 2 \mathrm{O}$ group. The right sub-picture shows dramatic planar color changes with time indicative of the planar snow depth variations, and these correspond well to the snow depth changes in the left sub-picture. 
Moreover, scatterplots of snow depth retrievals by the PBO H2O group and this work versus the snow depth measured by SNOTEL are provided in Fig. 8. The calculated root mean square errors (RMSEs) and correlation coefficients between the SNOTEL and GPS method data are shown in Table 1.

The proposed method produced an improvement in snow depth retrieval accuracy (RMSE: $7.40 \mathrm{~cm}$, Corr.: 0.99) compared to the PBO H2O group calculation results (RMSE: $16.58 \mathrm{~cm}$, Corr.: 0.99). Moreover, the grid diagram of the snow depth not only helped with the calculation of snow depth at the station, but it also reflected the snow depth distribution of the corresponding area.

\section{Discussion}

At present, global navigation satellite systems (GNSSs) available for use include the GPS, GLONASS, GALILEO, and BDS, all of which are continually developing both in terms of satellite data quantities and frequency bands. Multi-satellites and multi-frequencies means that more reflected signals are available, which will benefit further research of multi-GNSS reflected signals.

Only the geometric delay in the phase difference between the direct and the reflected signals has been considered, and phase terms caused by the composition of the surface material have been neglected.

\section{Acknowledgments}

Some of this material was based on data, equipment, and engineering services provided by the Plate Boundary Observatory operated by UNAVCO for EarthScope (http://www.earthscope.org) and by the PBO H2O research group

(http://xenon.colorado.edu/portal/). SNOTEL data shown in this paper were retrieved from http://www.wcc.nrcs.usda.gov/. This work was supported by the National Science Foundation of China (41104019, 41274005, and GFZX0301040308). We would like to thank the anonymous reviewers for their valuable comments and suggestions. We also thank Editage (www.editage.cn) for English language editing.

\section{References}

Bilich, A., Larson, K.M., Axelrad, P., 2004. Observations of signal-to-noise ratios (SNR) at geodetic GPS site CASA: Implications for phase multipath.

Proceedings of the Centre for European Geodynamics and Seismology 23, 77-83.

Boniface, K., Braun, J.J., McCreight, J.L., Nievinski, F.G., 2015. Comparison of snow data assimilation system with GPS reflectometry snow depth in the western United States. Hydrol. Process. 29(10), 2425-2437.

Grant, M.S., Acton, S.T., Katzberg, S.J., 2007. Terrain moisture classification using GPS surface-reflected signals. IEEE Geosci. Remote Sens. Lett. 4(1), 41-45. 
Hall, C.D., Cordey, R.A., 1988. Multistatic scatterometry. Proceedings of IGARSS '88 Symposium, pp. 561-562.

Hristov, H.D., 2000. Fresnel Zones in Wireless Links, Zone Plate Lenses and Antennas. Artech House, ISBN 978-0890068496, 345 pp.

Jacobson, M.D., 2010. Snow-covered lake ice in GPS multipath reception - Theory and measurement. Adv. Space Res. 46(2), 221-227.

Jin, S., Najibi, N., 2014. Sensing snow height and surface temperature variations in Greenland from GPS reflected signals. Adv. Space Res. 53(11), 1623-1633.

Katzberg, S.J., Torres, O., Grant, M.S., Masters, D., 2006. Utilizing calibrated GPS reflected signals to estimate soil reflectivity and dielectric constant: results from SMEX02. Remote Sens. Environ. 100(1), 17-28.

Larson, K.M., Braun, J.J., Small, E.E, Zavorotny, V.U, Gutmann, E.D, Bilich, A.L, 2010. GPS multipath and its relation to near-surface soil moisture content. IEEE J. Sel. Top. Appl. Earth Obs. Remote Sens. 3(1), 91-99.

Larson, K.M., Gutmann, E.D., Zavorotny, V.U., Braun, J.J., Williams, M.W., Nievinski, F.G., 2009. Can we measure snow depth with GPS receivers? Geophys. Res. Lett. 36(17), 1-5.

Larson, K.M., Nievinski, F.G., 2013. GPS snow sensing: results from the EarthScope Plate Boundary Observatory. GPS Solut. 17(1), 41-52.

Larson, K.M., Small, E.E., Gutmann, E., Bilich, A., Axelrad, P., Braun, J., 2008 a. Using GPS multipath to measure soil moisture fluctuations: initial results. GPS Solut. 12(3), 173-177.

Larson, K.M., Small, E.E., Gutmann, E., Bilich, A., Braun, J., Zavorotny, V.U., 2008b. Use of GPS receivers as a soil moisture network for water cycle studies. Geophys. Res. Lett. 35, L24405, doi:10.1029/2008GL036013.

Nievinski, F.G., 2013. Forward and inverse modeling of GPS multipath for snow monitoring. Ph.D. Dissertation, University of Colorado at Boulder.

Nievinski, F.G., Larson K.M., 2014a. Inverse modeling of GPS multipath for snow depth estimation-Part I: Formulation and simulations. IEEE Trans. Geosci. Remote Sens. 52(10), 6555-6563.

Nievinski, F.G., Larson, K.M., 2014b. Inverse modeling of GPS multipath for snow depth estimation-Part II: Application and validation. IEEE Trans. Geosci. Remote Sens. 52(10), 6564-6573.

Ozeki, M., Heki, K., 2011. GPS snow depth meter with geometry-free linear combinations of carrier phases. J. Geodesy 86(3), 209-219.

Rodriguez-Alvarez, N., Aguasca, A., Valencia, E., Bosch-Lluis, X., Camps, A., Ramos-Perez, I., Park, H., Vall-llossera, M., 2012a. Snow thickness monitoring using GNSS measurements. IEEE Geosci. Remote Sens. Lett. 9(6), 1109-1113.

Rodriguez-Alvarez, N., Bosch-Lluis, X., Camps, A., Ramos-Perez, I., Valencia, E., Park, H., Vall-llossera, M., 2012b. Vegetation water content estimation using GNSS measurements. IEEE Geosci. Remote Sens. Lett. 9(2), 282-286.

Serreze, M.C., Clark, M.P., Armstrong, R.L., McGinnis, D.A., Pulwarty, R.S., 1999. Characteristics of the western United States snowpack from snowpack telemetry (SNOTEL) data. Water Resour. Res. 35(7), 2145-2160. 
Yu, K., Ban, W., Zhang, X., Yu, X., 2015. Snow depth estimation based on multipath phase combination of GPS triple-frequency signals. IEEE Trans. Geosci. Remote Sens. 53(9), 1-10.

Zavorotny, V.U., Larson, K.M., Braun, J.J., Small, E.E., Gutmann, E.D., Bilich, A.L., 2010. A physical model for GPS multipath caused by land reflections: Toward bare soil moisture retrievals. IEEE J. Sel. Top. Appl. Earth Obs. Remote Sens. 3(1), 100-110. 